SFB

Tone onset detection using an

823

auditory model

Nadja Bauer, Klaus Friedrichs,

Dominik Kirchhoff, Julia Schiffner, Claus Weihs

Nr. 50/2012

$\infty$

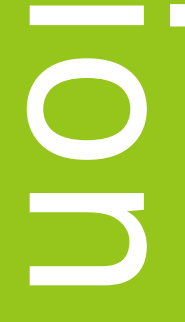

0

(1)

(O)

(D)

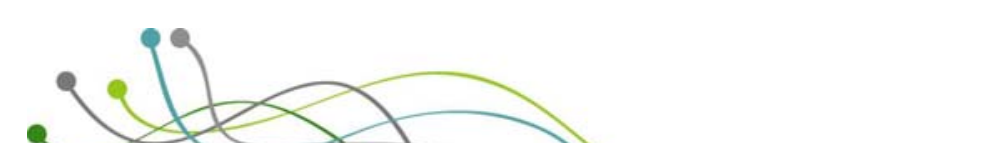





\title{
Tone Onset Detection Using an Auditory Model
}

Nadja Bauer, Klaus Friedrichs, Dominik Kirchhoff, Julia Schiffner and Claus Weihs

Chair of Computational Statistics, Faculty of Statistics, TU Dortmund

\{bauer,friedrichs, kirchhoff, schiffner, weihs\}

estatistik.tu-dortmund.de

\begin{abstract}
Onset detection is an important step for music transcription and other tasks frequently encountered in music processing. Although several approaches have been developed for this task, neither of them works well under all circumstances. In Bauer et al. (2012) we investigated the influence of several factors like instrumentation on the accuracy of onset detection. In this work, this investigation is extended by a computational model of the human auditory periphery. Instead of the original signal the output of the simulated auditory nerve fibers is used. The main challenge here is combining the outputs of all auditory nerve fibers to one feature for onset detection. Different approaches are presented and compared. Our investigation shows that using the auditory model output leads to essential improvements of the onset detection rate for some instruments compared to previous results.
\end{abstract}

\section{Introduction}

A tone onset is the time point of the beginning of a musical note or other sound. A tutorial on basic onset detection approaches is given by Bello et al. (2005). The algorithm we will use here is based on two approaches proposed in Bauer et al. (2012): In the first approach the amplitude slope and in the second approach the change of the spectral structure of an audio signal are considered as indicators for tone onsets. In Section 2 we briefly describe these two approaches.

Since the human ear still is the best system for onset detection the onset detection approach is extended by using the output of an auditory model instead of the original signal. A similar idea was proposed in Benetos et al. (2009) where the extension led to improved results. In contrast to our work in 
their study two other features are used for onset detection (spectral flux and group delay). Furthermore, they use fixed parameter settings for the detection algorithm while the goal of our study is to explore different settings by an experimental design.

Section 3 describes the auditory model utilized for this study. As the output is a multidimensional time series the onset detection indicators of all dimensions have to be combined to one indicator. Therefore, in Section 4 we propose several combination strategies.

To test our approach we use randomly generated tone sequences of six music instruments with two tempo settings. Section 5 provides the detailed generation procedure. Section 6 presents the results in respect of the music instruments, the tempo and the chosen combination strategy. Additionally, the results of the extended approach are compared to the results of the approach based on the original signal proposed in Bauer et al. (2012). Finally, Section 7 summarizes the work and provides ideas for future research.

\section{Onset Detection Approach}

The ongoing audio signal is split up into $T$ windows of length $\boldsymbol{L}$ samples with an overlap of $\boldsymbol{O}$ per cent of $\boldsymbol{L}$. In each window (starting with the second) two features are evaluated: The difference between amplitude maxima $\left(F_{A}\right)$ and the correlation coefficient between the spectra $\left(F_{S}\right)$ of the current and the previous window, respectively. Each of the vectors $F_{A}$ and $F_{S}$ is then rescaled into the interval $[0,1]$.

For each window a combined feature $C o m b F$ can be calculated as $C o m b F=$ $\boldsymbol{W}_{\boldsymbol{A}} \cdot F_{A}+\left(1-\boldsymbol{W}_{\boldsymbol{A}}\right) \cdot F_{S}$, where the weight $\boldsymbol{W}_{\boldsymbol{A}} \in[0,1]$ is a further parameter, which specifies the influence of the amplitude based feature on the weighted sum. In Bauer et al. (2012) we investigated further combination approaches and this approach provided the best results. Let $\operatorname{detect}\left(s, \boldsymbol{L}, \boldsymbol{O}, \boldsymbol{W}_{\boldsymbol{A}}\right)$ denote the onset detection function which returns the $C o m b F$-vector for the audio signal $s$ depending on $\boldsymbol{L}, \boldsymbol{O}$ and $\boldsymbol{W}_{\boldsymbol{A}}$.

In the following, we describe the peak-picking procedure, which consists of two steps: thresholding of the onset detection function and localization of tone onsets. In order to assess if a window contains a tone onset, based on $C o m b F$, a threshold is required. We will use here a $Q \%$-quantile of the $C o m b F$-vector as such threshold. If the $C o m b F$-value for the current window, but neither for the preceding nor for the succeeding window, exceeds the threshold, an onset is detected in this window. If the threshold is exceeded 
in multiple, consecutive windows, we assume that there is only one onset, located in that window with the maximal CombF-value in this sequence. This is a simple fixed thresholding approach, but as we consider only music signals with little dynamics, it is assumed to be appropriate here (see Bello et al. (2005)). For each window with an onset detected its beginning and ending time points are calculated and the onset time is then estimated by the centre of this time interval. Let onsets $(\mathrm{CombF}, \boldsymbol{Q})$ denote the onset estimation function which returns for the onset detection feature $\operatorname{CombF}=\operatorname{detect}\left(s, \boldsymbol{L}, \boldsymbol{O}, \boldsymbol{W}_{\boldsymbol{A}}\right)$ and quantile $\boldsymbol{Q}$ the estimated time points of the tone onsets.

In this work we assume that a tone onset is correctly detected, if the absolute difference between the true and the estimated onset time is less than $50 \mathrm{~ms}$ (see Dixon (2006)). As quality criterion for the goodness of the onset detection the so called $F$-value is used here:

$$
F=\frac{2 c}{2 c+f^{+}+f^{-}},
$$

where $c$ is the number of correctly detected onsets, $f^{+}$is the number of false detections and $f^{-}$denotes the number of undetected onsets (Dixon (2006)). Note that the $F$-value always lies between 0 and 1 . The optimal $F$-value is 1 . For the given vector of the true onset time points true, the function $f$ _value (est,true) returns the $F$-value for the vector of the estimated onset times est $=$ onsets $(\mathrm{CombF}, \boldsymbol{Q})$.

\section{Auditory Model}

An auditory model is a computer model of the human auditory system. It requires an acoustic signal as input and outputs the spike firing rates of the auditory nerve fibers. The human auditory system consists of roughly 3000 auditory nerve fibers. Auditory models contain usually a much smaller number of fibers. For this study the popular model of Meddis (2006) is employed. To put it simply, in this model the auditory system is coded by a multichannel bandpass filter where each channel represents one specific nerve fiber. Each channel has its specific best frequency by which its perceptible frequency range is defined. In this work we use 40 channels with best frequencies between $250 \mathrm{~Hz}$ (channel 1) and $7500 \mathrm{~Hz}$ (channel 40). An exemplary output of the model, called auditory image, can be seen in Figure 1. While the 40 channels are located on the vertical axis and the time response on the horizontal axis, the grey level indicates the channel activity per second. 


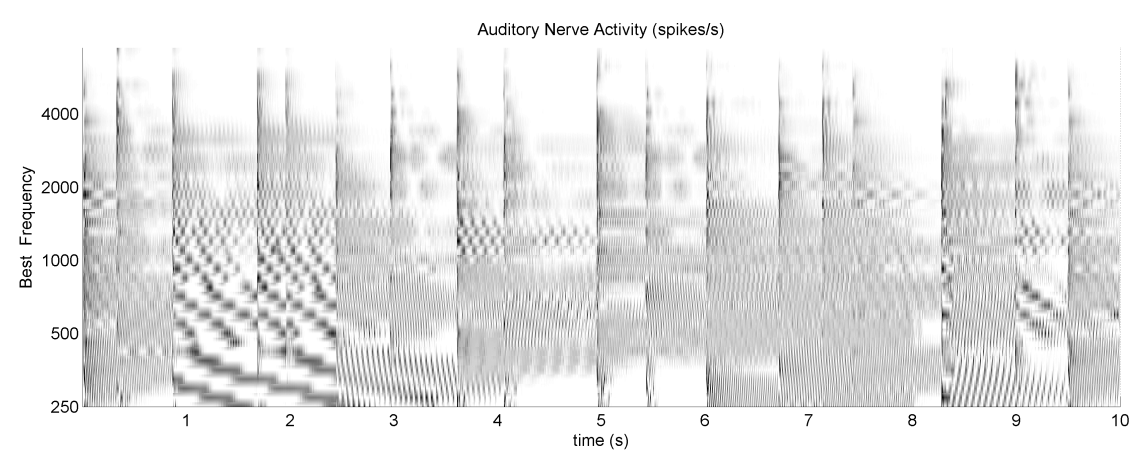

Fig. 1. Auditory image

\section{Auditory Model Based Onset Detection Approach}

The output of the auditory model is, according to Section 3, a set of 40 audio signals $\left(\operatorname{chan}_{1}, \operatorname{chan}_{2}, \ldots\right.$, chan $\left._{40}\right)$, where each signal corresponds to one of 40 hearing channels. For all channels the combined features CombF are computed as described in Section 2 and then combined to one onset indicator. While there are more comprehensive ways, we tried a simple method first. The detailed procedure is described in the following ${ }^{1}$ :

1 For each channel chan $_{k}, k=1, \ldots, 40$, calculate the onset detection function $\operatorname{CombF}_{k}=\operatorname{detect}\left(\operatorname{chan}_{k}, \boldsymbol{L}, \boldsymbol{O}, \boldsymbol{W}_{\boldsymbol{A}}\right)$.

2 For each window $i, i=1, \ldots, T$, do:

- $v[i]=\left(\operatorname{CombF}_{1}[i], \ldots, \operatorname{CombF}_{40}[i]\right)^{\prime}$,

- output $[i]=$ quantile $(v[i], \boldsymbol{C})$, where the function quantile returns the $C \%$-quantile of the vector $v[i]$.

3 output $=(\text { output }[1], \ldots, \text { output }[T])^{\prime}$.

4 est $=$ onsets $($ Output, $\boldsymbol{Q})$.

5 error $=f \_$value $($est, true $)$.

In the following we will investigate the influence on the onset detection accuracy of the parameters $\boldsymbol{L}, \boldsymbol{O}, \boldsymbol{W}_{\boldsymbol{A}}$ and $\boldsymbol{C}$. In order to reduce the complexity, we estimate the parameter $\boldsymbol{Q}$ by means of grid search in the interval from 0 to 1 with step size 0.05 (the same approach as in Bauer at al. (2012)).

The following settings of the four parameters are tested: $\boldsymbol{L}$ with two levels (512 and 2024 samples), $\boldsymbol{O}$ with two levels ( 0 and 50 per cent), $\boldsymbol{W}_{\boldsymbol{A}}$ with three levels ( $0,0.5$ and 1$)$ and $\boldsymbol{C}$ with three levels (5, 50 and 95 per cent). Table 1 lists the 36 parameter settings.

\footnotetext{
${ }^{1} v[i]$ is the notation for the $i$-th element of vector $v$.
} 
Table 1. Parameter settings for auditory model based onset detection

\begin{tabular}{|c|c|c|c|c|c|c|c|c|c|c|c|c|c|c|c|}
\hline & $L$ & $\boldsymbol{U}$ & ${ }_{A} C$ & & $L$ & $O$ & $W_{A} C$ & 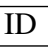 & $L$ & $O$ & $W_{A} C$ & - & $L$ & $O$ & \\
\hline & 51 & 0 & 0 & & & 50 & 0 & & & S & 8 & 28 & 8 & 50 & 5 \\
\hline & & & & & & & & & & & & & & & \\
\hline & & 0 & 80 & & & 50 & 95 & & & & & & & & \\
\hline & & 0 & & & & 5 & & & & 0 & & & & & \\
\hline & & 0 & & & & 50 & & & & & & & & & \\
\hline & & 0 & & & & 50 & & & & 0 & & & & & \\
\hline & & 0 & & & & 5 & & & & & & & & & 1 \\
\hline & & 0 & 1 & & & 50 & & & & & & 35 & & & 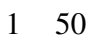 \\
\hline & 2 & 0 & 95 & 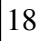 & 512 & 50 & 95 & 27 & 2048 & 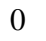 & 95 & 36 & 2048 & 50 & 95 \\
\hline
\end{tabular}

\section{Music Data Set}

In order to compare the original onset detection with the extended approach proposed in this paper the same data set is used as in Bauer et al. (2012), which consists of 24 randomly generated tone sequences. In the following we briefly describe the construction principles of this data set.

There are many characteristics that describe a music signal like tempo, genre, instrumentation or sound volume. We consider merely the instrumentation and the tempo as control variables when designing the data set: The same tone sequences are recorded by different music instruments with different tempo settings. This allows to explicitly measure the influence of these two control variables on the accuracy of the onset detection.

Six music instruments are considered: piano, guitar, flute, clarinet, trumpet and violin. The tone sequences are generated randomly by considering the following settings:

1. Sound intensities follow the uniform distribution in the interval $[70,90]$ (in MIDI-coding).

2. Notes follow the uniform distribution in the interval [60,76] (in MIDIcoding). This interval corresponds to the common pitch range (from $\mathrm{C} 4$ to E5) of the instruments under consideration.

3. Tone durations (in seconds) are

a) absolute values from the normal distribution with parameters $\mu=0.5$ and $\sigma=0.2$,

b) absolute values from the normal distribution with parameters $\mu=0.2$ and $\sigma=0.1$.

The first tone duration setting generates slow music pieces with two beats per second on average (or $120 \mathrm{BPM}^{2}$ ), whereas the second setting gener-

\footnotetext{
2 BPM: beats per minute
} 
ates fast music pieces with five beats per second on average (300 BPM). For each of six music instruments and for each of two tone duration settings five tone sequences (with the duration of 10 seconds) are generated. The fast tone sequences hence include ca. 50 tone onsets and the slow sequences ca. 20 onsets.

The generated MIDI-files are converted to WAVE-files using real tone samples from the RWC data base (Goto et al. (2003)).

\section{Results}

In this section we will compare the 36 proposed parameter settings of the auditory model based onset detection in order to investigate the influence of these settings on the goodness of onset detection for each instrument. Further, we will compare the results of original signal and auditory model based detection for the same algorithm's parameter settings. This should reveal whether an extension of the onset detection algorithm (proposed in our previous work) with an auditory model leads to better detection accuracy.

\subsection{Comparison of Auditory Image Based Onset Detection Approaches}

In order to compare the goodness of the proposed 36 onset detection strategies (see Section 4) we calculate the $F$-measure for each music instrument and for each of the five slow and five fast sequences (see Section 5). The resulting average $F$-measures of the six music instruments and 36 strategies are presented in Figure 2. Most strategies provide acceptable results but few strategies obviously fail. Furthermore, it can be seen, that for the music instruments piano, guitar and clarinet - in contrast to the others - systematically better detection accuracies are achieved.

Table 2 presents the best parameter settings for each instrument and on average. We can observe an interaction between parameters $\boldsymbol{C}$ and $\boldsymbol{W}_{\boldsymbol{A}}$ : if $\boldsymbol{W}_{\boldsymbol{A}}=1, \boldsymbol{C}$ should be chosen high $(\boldsymbol{C}=95)$. Whereas the best setting on average is the one with the small window length $(\boldsymbol{L}=512$ samples), without overlap $(\boldsymbol{O}=0 \%)$, considering merely the spectral based feature $\left(\boldsymbol{W}_{\boldsymbol{A}}=0\right)$ and middle quantile (median, $\boldsymbol{C}=50 \%$ ). Further, in Table 3 the worst strategies are listed: Considering only the amplitude based feature $\left(\boldsymbol{W}_{\boldsymbol{A}}=1\right)$ in combination with small $\boldsymbol{C}$-values does not seem to lead to acceptable results. 


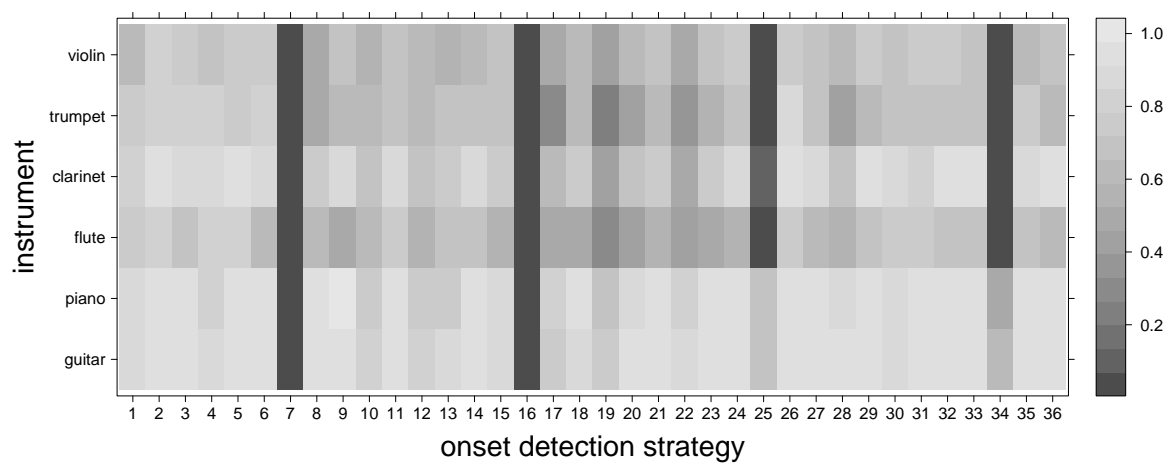

Fig. 2. Comparison of the 36 onset strategies (see Table 1): For each instrument and each strategy the average $F$-value over five slow and five fast sequences is presented

Table 2. Best parameter settings for each instrument and on average

\begin{tabular}{|llllll|}
\hline instrument & ID & $\boldsymbol{L}$ & $\boldsymbol{O}$ & $\boldsymbol{W}_{\boldsymbol{A}}$ & $\boldsymbol{C}$ \\
\hline piano & 36 & 2048 & 50 & 1 & 95 \\
guitar & 23 & 2048 & 0 & 0.5 & 50 \\
flute & 9 & 512 & 0 & 1 & 95 \\
clarinet & 36 & 2048 & 50 & 1 & 95 \\
trumpet & 2 & 512 & 0 & 0 & 50 \\
violin & 2 & 512 & 0 & 0 & 50 \\
\hline on average & 2 & 512 & 0 & 0 & 50 \\
\hline
\end{tabular}

Table 3. Worst parameter settings

\begin{tabular}{|lllll|}
\hline ID & $\boldsymbol{L}$ & $\boldsymbol{O}$ & $\boldsymbol{W}_{\boldsymbol{A}}$ & $\boldsymbol{C}$ \\
\hline 7 & 512 & 0 & 1 & 5 \\
16 & 512 & 50 & 1 & 5 \\
34 & 2048 & 50 & 1 & 5 \\
25 & 2048 & 0 & 1 & 5 \\
19 & 2048 & 0 & 0 & 5 \\
\hline
\end{tabular}

\subsection{Comparison of Auditory Image Based and Original Signal Based Onset Detection}

In Bauer et al. (2012) the original signal based tone onset detection algorithm was tested on the data set used in this work for eight possible combinations of the following parameter settings: $\boldsymbol{L}$ with two levels (512 and 2048 samples), $\boldsymbol{O}$ with two levels (0\% and 50\%) and $\boldsymbol{W}_{\boldsymbol{A}}$ with two levels (0 and 1). As the parameter $\boldsymbol{C}$ is only given in the extended approach, it was set to the average best setting $\boldsymbol{C}=50 \%$ (see Table 2).

Table 4 shows the average $F$-values of the auditory model based and original signal based onset detection over the eight parameter settings mentioned above. It also gives the median of the percentage change of the results when using the extended approach. Further, Figure 3 compares the detection accuracy $(F$-measure) of both approaches for an example of slow trumpet tone sequences. 
According to Table 4 the auditory image based onset detection is on average (over all parameter settings) better than the original signal based. However, when considering the median of the vector of 40 improvement-rates for each setting and each tone sequence - we observe for flute and trumpet in contrast to other music instruments - decline of the onset detection accuracy when using the auditory model based approach (especially for the slow sequences). This is basically caused by the fact that many onsets for these two instruments were detected with a delay of more than $50 \mathrm{msec}$. Figure 4 shows a fragment of the auditory image for a slow trumpet sequence. Obviously the channels with high best frequencies (over $3000 \mathrm{~Hz}$ ) respond later than the channels with low best frequencies. Interestingly such delays do not occur very often for short tones from staccato tone libraries (for trumpet and flute). This may be due to the fact that short and especially staccato tones in contrast to long tones usually have stronger attacks. Thus, in order to improve the onset detection a systematic investigation of these effects is required.

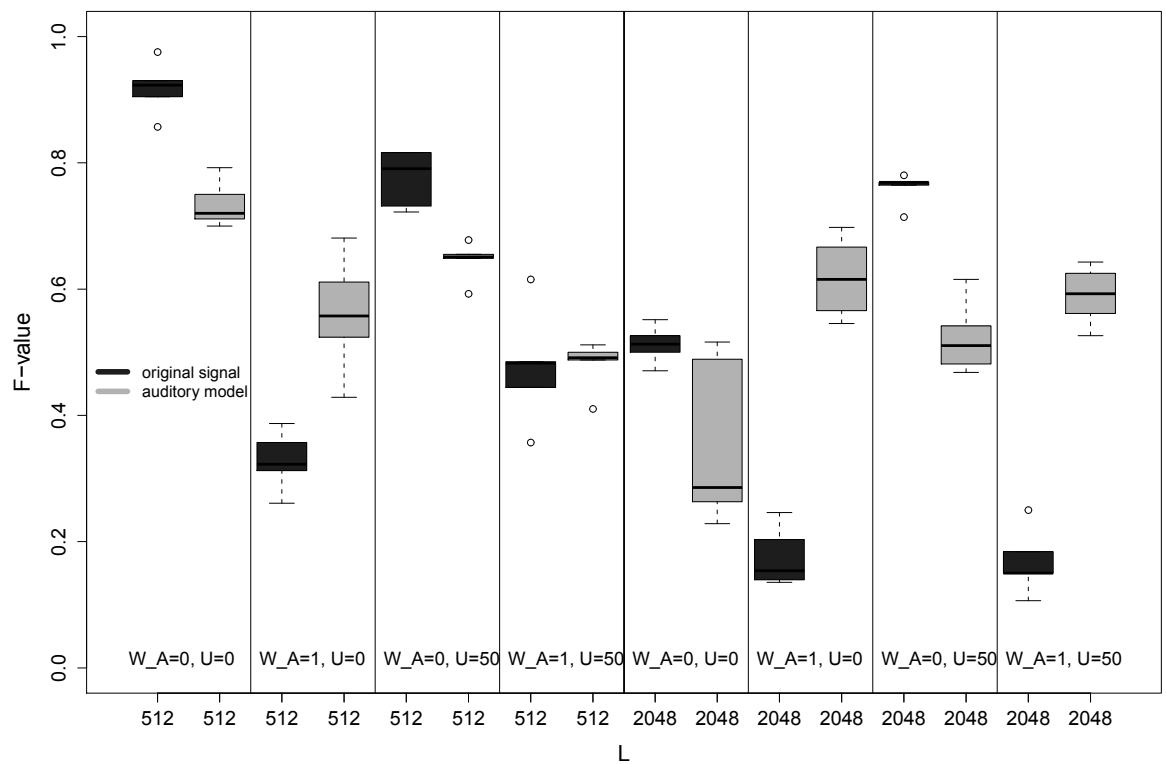

Fig. 3. Comparison of $F$-values of the original signal and auditory image based onset detection for slow trumpet tone sequences 
Table 4. Averaged $F$-values of the auditory model and original signal based onset detection over eight different parameter settings and the median of the percentage change of the results when using the extended approach

\begin{tabular}{|l|llllll|}
\hline & \multicolumn{7}{|c|}{ sequence } \\
\hline approach & piano & piano & guitar & guitar & flute \\
slow & fast & slow & $\begin{array}{l}\text { flute } \\
\text { fast }\end{array}$ \\
\hline auditory model & 0.961 & 0.934 & 0.950 & 0.928 & 0.576 & 0.662 \\
original signal & 0.918 & 0.854 & 0.949 & 0.895 & 0.553 & 0.606 \\
improvement (in \%) & 1.33 & 5.75 & 0.00 & -0.98 & -6.73 & -1.64 \\
\hline approach & clarinet & clarinet & trumpet & trumpet & violin & violin \\
& llow & fast & slow & fast & slow & fast \\
\hline auditory model & 0.849 & 0.875 & 0.564 & 0.708 & 0.597 & 0.773 \\
original signal & 0.775 & 0.801 & 0.514 & 0.658 & 0.466 & 0.638 \\
improvement (in \%) & 4.53 & 3.95 & -6.78 & -0.46 & 32.56 & 23.92 \\
\hline
\end{tabular}

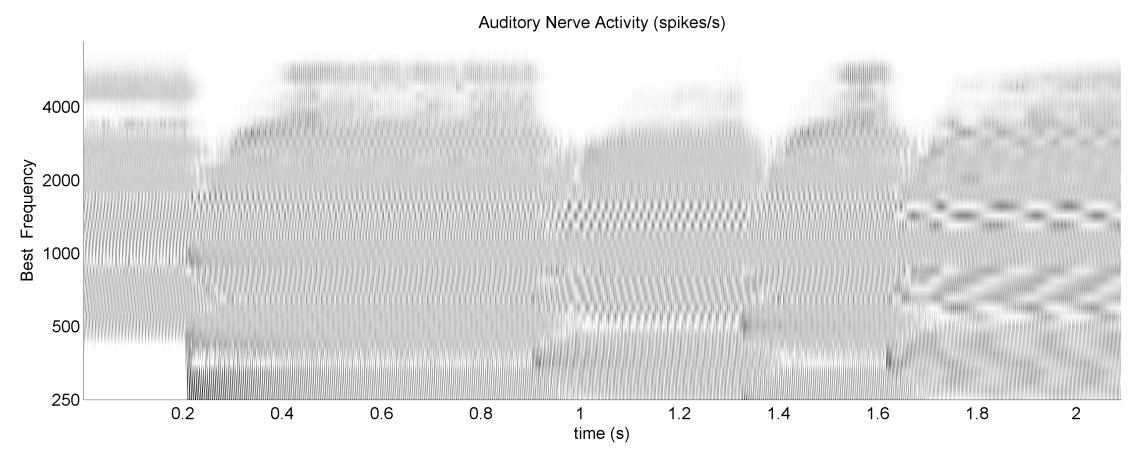

Fig. 4. Cut-out of the auditory image for a slow trumpet sequence

\section{Summary}

In this work we proposed a strategy for using the multiple channel output of an auditory model for the tone onset detection problem. We compared the auditory model and original signal based approaches on the same data set for several algorithm parameter settings. The data set consists of random sequences which were generated for six music instruments and two tempi (fast and slow) using real tone recordings.

An essential improvement of the onset detection when using the auditory model can be noted for the violin (over $20 \%$ ). However, for the slow 
trumpet and flute tone sequences a significant decline of the detection accuracy can be observed using the auditory model based approach. This can be explained through the delayed response of high frequency channels of the auditory model. In order to improve the auditory model based onset detection the detailed study of auditory model behavior depending on musical characteristics of audio signals is required and is the aim for our further research. Additionally, we plan to compare the features we used for onset detection (amplitude difference and correlation between spectra) with the ones used by Benetos et al. (2009) and to develop a strategy for combining these features. Further, other approaches for processing the channel outputs of the auditory model can be considered, for example by taking the channel order into account.

\section{Acknowledgements}

This work was supported by the Collaborative Research Centre "Statistical Modelling of Nonlinear Dynamic Processes" (SFB 823) of the German Research Foundation (DFG), within the framework of Project C2 "Experimental Designs for Dynamic Processes" and Project B3 "Statistical Modelling of Highly-Resolved Spectro-Temporal Audio Data in Hearing Aids".

\section{References}

BAUER, N., SCHIFFNER, J. and WEIHS, C. (2012): Einfluss der Musikinstrumente auf die Güte der Einsatzzeiterkennung. Discussion Paper 10/2012. SFB 823, TU Dortmund

BELLO, J. P., DAUDET, L., ABDALLAH, S., DUXBURY, C., DAVIES, M. and SANDLER, M. B. (2005): A Tutorial on Onset Detection in Music Signals. IEEE Transactions on Speech and Audio Processing, 13 (5), 1035-1047.

DIXON, S. (2006): Onset detection revisited. In: Proceedings of the 9th International Conference on Digital Audio Effects (DAFx-06), 133-137.

BENETOS, E., HOLZAPFEL, A., STYLIANOU Y. (2009): Pitched instrument onset detection based on auditory spectra. 10th International Society for Music Information Retrieval Conference (ISMIR 2009), 105-110.

MEDDIS, R (2006): Auditory-nerve first-spike latency and auditory absolute threshold: A computer model. Journal of the Acoustical Society of America, 116, 406-417.

GOTO, M., HASHIGUCHI, H., NISHIMURA, T., and OKA, R. (2003): RWC Music Database: Music Genre Database and Musical Instrument Sound Database, Proceedings of the 4th International Conference on Music Information Retrieval (ISMIR 2003), 229 230. 

